\title{
Guillain-Barré Syndrome in a Patient with Hereditary Spastic Paraparesis
}

\author{
A Case Report \\ Jassim Y. Alhashel ${ }^{a}$ Asmahan F. Alshubailia Ashraf Montasser ${ }^{a}$ T. Anim ${ }^{b}$ \\ aDepartment of Neurology, Ibn Sina Hospital, and bDepartment of Pathology, Faculty of Medicine, \\ Kuwait University, Kuwait
}

\author{
Key Words \\ Guillain-Barré syndrome $\cdot$ Hereditary spastic \\ paraparesis $\cdot$ Henoch-Schönlein purpura
}

quired treatable causes of weakness in such patients in order to prevent an increase in disability.

Copyright $@ 2004$ S. Karger AG, Basel

\begin{abstract}
Objective: To report the unusual occurrence of GuillainBarré syndrome (GBS) in a case of hereditary spastic paraparesis (HSP) and describe its effect on the preexisting clinical picture as well as the importance of early recognition and treatment. Clinical Presentation and Intervention: A 41-year-old man known to have HSP developed an acute and rapid deterioration of muscle power associated with paresthesia in both upper and lower limbs. Clinical examination revealed flaccid quadriparesis with areflexia. Nerve conduction studies were suggestive of demyelinating polyneuropathy and CSF analysis showed increased protein but no cells. The diagnosis of GBS was made in addition to HSP. The patient received intravenous immunoglobulins $20 \mathrm{mg} / \mathrm{kg}$ body weight/day for 5 days. He responded very well to the treatment clinically and neurophysiologically and thereafter, he reverted to the preexisting spastic picture of HSP. Conclusion: The peripheral demyelination with GBS was severe enough to obscure the preexisting clinical picture, but fortunately it responded very well to treatment. The clinical associations with HSP are widely variable, therefore it is very important to recognize ac-
\end{abstract}

\section{Introduction}

Hereditary spastic paraparesis (HSP) is a disorder characterized by progressive lower limb weakness and spasticity that is more striking than muscular weakness. The age of onset is variable and the condition has a relatively benign course. In complicated forms of HSP, cerebellar signs, pes cavus, wasted hands, optic atrophy and peripheral neuropathy have been reported [1].

In this paper, we report a case of a 41-year-old male patient with a pure form of HSP who developed an acute inflammatory demyelinating polyradiculopathy (Guillain-Barré syndrome, BGS) during the course of illness. Its impact on the clinical picture, diagnosis and management are described. No similar case has been described in the literature.

\section{Case Report}

A 41-year-old Kuwaiti male patient presented with a 3-year history of slowly progressive lower limb weakness and stiffness when walking. He had no involvement of the upper limbs, visual complaints, sensory disturbances, cognitive changes, imbalance or

\begin{tabular}{ll}
\hline KARGER & (c) 2004 S. Karger AG, Basel \\
Fax +4161306 1234 & $1011-7571 / 04 / 0136-0369 \$ 21.00 / 0$ \\
$\begin{array}{l}\text { E-Mail karger@karger.ch } \\
\text { www.karger.com }\end{array}$ & $\begin{array}{l}\text { Accessible online at: } \\
\text { www.karger.com/mpp }\end{array}$
\end{tabular}

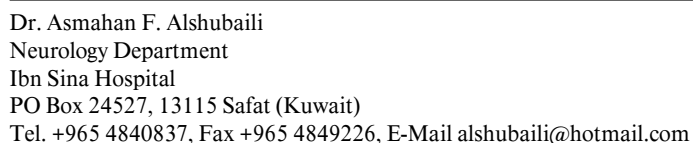


Fig. 1. Electron microscopy showing degenerative myelin of large myelinated fibers with associated axonal sprouting (A). Note that there is increased endoneural collagen $(\mathrm{C})$.

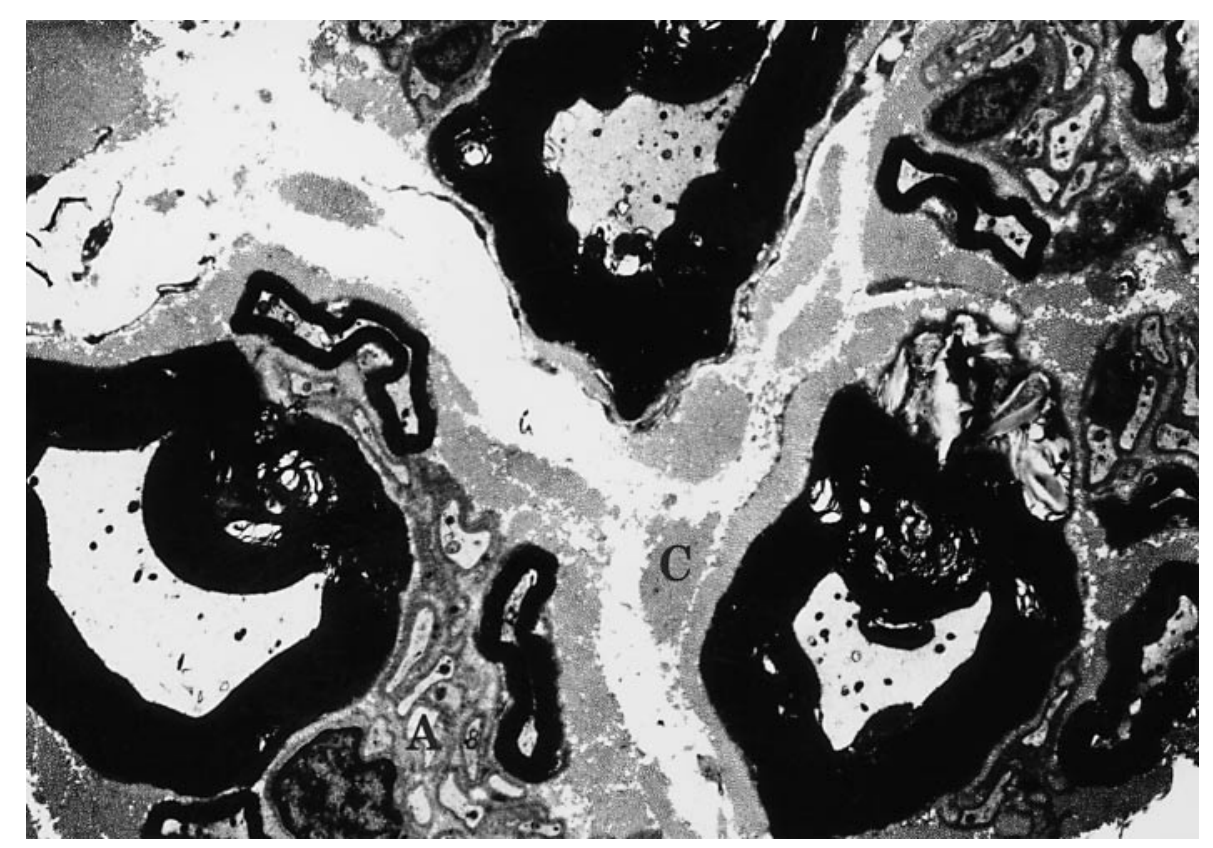

sphincter involvement. He had a 34-year-old brother, who had an identical problem, but his 4 other brothers and 1 sister were normal. No other family member was involved and his parents are second cousins. Physical examination revealed spastic paraparesis with grade 4/5 weakness, hyperreflexia, clonus of both ankle and knee jerks and upgoing plantar reflexes. Fundi were normal and there was no clinical evidence of sensory deficit, muscle wasting or cerebellar signs and his gait was spastic. The 34-year-old sibling also showed identical findings. Nerve conduction studies were normal. The clinical picture was suggestive of HSP. Eighteen months after diagnosis, the patient was admitted to hospital with a 2-week history of acute onset of ascending paresthesia involving both upper and lower limbs and increasing muscle weakness of the lower limbs. There was no facial or bulbar muscle weakness. He could not walk unaided. There was no clear history of a flu-like illness prior to the illness.

Clinical examination revealed normal higher mental functions, high-arched palate, normal fundi, full extraocular movements, normal visual acuity and no facial weakness. There was flaccid weakness of both upper and lower limbs with grade $4 / 5$ muscle power in the upper limbs and $3 / 5$ proximally and distally in the lower limbs.

There was total areflexia of all four limbs and plantar reflexes were absent. No sensory deficit was detected despite his sensory complaint, and he was unable to stand on his feet unsupported.

Laboratory investigations showed normal biochemical profile and complete blood count and erythrocyte sedimentation rate of $6 \mathrm{~mm} / \mathrm{h}$. Thyroid function tests, folate and $B_{12}$ levels were normal. Brucella slide agglutination test, VDRL and TPHA tests were negative. HTLV I and III were negative in both serum and cerebrospinal fluid (CSF), and urine was negative for porphobilinogen. CSF study revealed no cells, but protein was 1,049 mg/l (normal 150-450 mg/l), sugar $3.8 \mathrm{mmol}$ (serum sugar level was 5.2). Oligoclonal bands were not detected.

Nerve conduction study disclosed absence of $\mathrm{H}$ reflex bilaterally, prolongation of terminal motor latencies and F-wave latencies, slow- ing of motor conduction of all tested nerves. All compound muscle action potentials were low in amplitude and dispersed with significant proximal conduction block particularly of right peroneal, left tibial and left ulnar nerves. Right median and left ulnar sensory nerve action potentials were low in amplitude, and sensory conduction velocities were slow. Findings were suggestive of inflammatory demyelinating polyneuropathy, a case of GBS superimposed on HSP. Sural nerve biopsy was done 4 weeks after onset. Light microscopy showed focal demyelination with accompanying axonal degeneration, scattered mononuclear infiltration of endoneurium, especially related to demyelinating foci and blood vessels with increased mast cells. Electron microscopy showed focal myelin degeneration, and axons showed degenerative vesicles and widespread axonal sprouting. No inflammatory infiltrates were seen (fig. 1).

The patient received a course of intravenous immunoglobulin ( $20 \mathrm{mg} / \mathrm{kg}$ body weight/day) for 5 days, carbamazepine for paresthesia and regular physiotherapy. The patient's paresthesia and weakness improved 5 weeks after the onset. The upper limb deep tendon reflexes reappeared, but areflexia persisted in the lower limbs; however, he was able to walk with some support. Six weeks later, the lower limb reflexes started to reappear and the plantar reflexes became upgoing and the tone increased. He was discharged 8 weeks after admission, walking unsupported.

On follow-up in the outpatient clinic, 2 months after discharge, the patient was still walking unsupported but with a spastic gait. He had reverted to the previous state of spastic paraparesis with muscle power of $4 / 5$ in the lower limbs and $5 / 5$ in the upper limbs. Nerve conduction studies showed significant improvement, but $\mathrm{H}$ reflex was still absent bilaterally. 


\section{Discussion}

HSP consists of a group of disorders characterized by an insidious onset of progressive, generally severe spastic paraparesis. The prime pathology involves axonal degeneration of the longest descending (corticospinal) and ascending tracts both crossed and uncrossed and fasciculus gracilis. The degeneration is maximal at the terminal portions of the corticospinal tract and the cervical part of the dorsal columns and is found in about $50 \%$ of patients [2, 3].

HSP may be transmitted as an X-linked, autosomal recessive or autosomal dominant trait. Over the years, the genetic heterogeneity of the disorder has become more evident with 17 different HSP loci identified so far [4]. The majority of these genetically different forms are indistinguishable clinically, thus making careful history taking and clinical examination and exclusion of treatable causes of spastic paraparesis of paramount importance.

In 'complicated' forms of HSP, several clinical associations have been reported including optic atrophy, dementia, ichthyosis, mental retardation, hydrocephalus, agenesis of corpus callosum, extrapyramidal manifestation, cerebellar ataxia, Troyer syndrome, dystonia, sensory neuropathy, and amyotrophy of small muscles $[2,5]$. Such patients should always be evaluated thoroughly for concurrent or alternative neurological disorders rather than accepting them easily as variants of HSP.

In our patient and his affected sibling, such clinical associations were not found clinically or subclinically by neurophysiologic tests and MRI, and therefore they most likely had a pure motor form of HSP. Thus, when the patient developed acute deterioration in muscle power, other complications had to be excluded.

The changes in nerve conduction studies and CSF were all suggestive of an acquired etiology characteristic of GBS. This was confirmed by the findings in the nerve biopsy, which demonstrated changes suggestive of demyelination, and sprouting indicating good prognosis.

GBS is the most frequent acquired acute inflammatory demyelinating polyneuropathy, characterized by an acute onset of peripheral and cranial nerve dysfunction. Viral respiratory or gastrointestinal infections, immunization, or surgery often precede neurological symptoms by 5 days to 3 weeks. None of these antecedents could be elicited in this patient. The symptoms are usually most severe in the first week of onset but may progress over 3 weeks or more, and the rate of recovery is variable. Predictors of outcome include the severity of clinical disability at presentation, need for intubation and cranial nerve involvement, which indicate poorer outcome. Neurophysiologically, a demyelinating type of GBS with conduction block, as in our patient, is indicative of a better prognosis, unlike axonal forms [6]. Clinically, the axonal variant is similar to the demyelinating form of GBS, but a more severe course, with frequent respiratory involvement, ventilator dependence and significant residue may be seen [7].

The early recognition and treatment of GBS with intravenous immunoglobulins or plasmapheresis accelerate recovery, which might otherwise be delayed in some patients for months. In untreated cases about 35\% of patients have permanent residual hyporeflexia, atrophy, and weakness of distal muscles or facial paresis.

In our patient, it is worthwhile noting that the degree of peripheral demyelination was severe enough to obscure the spasticity in a patient suffering from long-standing HSP. However, the patient had a very good response to treatment with intravenous immunoglobulins, which emphasizes the need to look carefully for acquired and treatable causes for increasing weakness in these patients. This good prognosis was predicted by neurophysiological and pathological results.

\section{Conclusion}

To our knowledge, this is the first report of GBS in a patient with HSP and it demonstrates the significance of identifying acquired treatable causes for clinical deterioration in this group of patients. Greater morbidity or lifethreatening situations, like respiratory failure in GBS, can be avoided if these conditions are recognized, monitored, and treated early in their course.

\footnotetext{
References $\quad 1$ Fink JK: Advances in hereditary spastic paraplegia. Curr Opin Neurol 1997:10:313-318.

-2 Fink JK, Hedera P: Hereditary spastic paraplegia: Genetic heterogeneity and genotype-phenotype correlation. Semin Neurol 1999;19: 301-309.

3 Bruyn RP: The neuropathology of hereditary spastic paraparesis. Clin Neurol Neurosurg 1992;94(suppl):S16-18.

4 Fink JK: Hereditary spastic paraplegia. Neurol Clin 2002;20:711-726.

5 Harding AE: Hereditary spastic paraplegias. Semin Neurol 1993;13:333-336.

6 Ammache Z, Afifi AK, Brown CK, Kimura J: Childhood Guillain-Barré syndrome: Clinical and electrophysiological features predictive of outcome. J Child Neurol 2001;16:47-83.

7 Chowdhury D, Arora A: Axonal Guillain-Barré syndrome: A critical review. Acta Neurol Scand 2001;103:267-277.
} 